\title{
Micafungin versus anidulafungin in critically ill patients with invasive candidiasis: a retrospective study
}

\author{
Patrick J. van der Geest ${ }^{1 *}$, Nicole G. M. Hunfeld ${ }^{1,2}$, Sophie E. Ladage ${ }^{1}$ and A. B. Johan Groeneveld
}

\begin{abstract}
Background: In critically ill patients the incidence of invasive fungal infections caused by Candida spp. has increased remarkably. Echinocandins are recommended as initial treatment for invasive fungal infections. The safety and efficacy of micafungin compared to caspofungin is similar, but no comparison is made between anidulafungin and micafungin concerning safety and efficacy. We therefore performed a retrospective study to assess these aspects in critically ill patients with invasive candidiasis.

Methods: All patients in the intensive care unit (ICU) with invasive candidiasis, who were only treated with anidulafungin or micafungin, between January 2012 and December 2014 were retrospectively included. Baseline demographic characteristics, infection characteristics and patient courses were assessed.

Results: A total of 63 patients received either anidulafungin $(n=30)$ or micafungin $(n=33)$ at the discretion of the attending intensivist. Baseline characteristics were comparable between the two groups, suggesting similar risk for developing invasive candidiasis. Patients with invasive candidiasis and liver failure were more often treated with anidulafungin than micafungin. Response rates were similar for both groups. No difference was observed in 28-day mortality, but 90-day mortality was higher in patients on anidulafungin. Multivariable cox regression analysis showed that age and serum bilirubin were the best parameters for the prediction of 90-day mortality, whereas APACHE II, Candida score and antifungal therapy did not contribute $(P>0.05)$. None of the patients developed impaired liver function related to antifungal use and no differences were seen in prothrombin time, serum transaminases and bilirubin levels between the groups, after exclusion of patients with liver injury or failure.

Conclusion: Micafungin can be safely and effectively used in critically ill patients with invasive candidiasis. The observed increased 90-day mortality with anidulafungin can be explained by intensivists unnecessarily avoiding micafungin in patients with liver injury and failure.
\end{abstract}

Keywords: Critically ill, Micafungin, Anidulafungin, Invasive candidiasis

\section{Background}

The incidence of invasive fungal infections caused by Candida spp. is increasing in critically ill patients $[1,2]$. The latter is associated with prolonged duration of hospitalization and higher mortality rates [3-5]. The mortality of invasive candidiasis is directly correlated with a delay in starting antifungal therapy and therefore

\footnotetext{
*Correspondence: p.vandergeest@erasmusmc.nl

'Department of Intensive Care Medicine, Erasmus University Medical Center,

's Gravendijkwal 230, 3015 CE Rotterdam, The Netherlands

Full list of author information is available at the end of the article
}

early treatment with an appropriate antifungal drug is mandatory $[6,7]$.

A relatively novel class of antifungal agents are echinocandins which have activity against a broad spectrum of Candida spp., including C. glabrata and C. krusei, against which fluconazole has less activity [8]. Echinocandins are recommended as initial treatment for invasive candidiasis in patients with moderate to severe illness, keeping fluconazole reserved for less critically ill patients [9]. The recommendation are based on prospective randomized clinical trials which demonstrated that all three echinocandins are at least as effective as fluconazole for the 
treatment of invasive candidiasis [10-15]. The antifungal activity, pharmacokinetics and toxicity profile of each echinocandin is slightly different, but the relevance of this finding remains unclear [16]. Both caspofungin and micafungin undergo hepatic metabolism, in contrast to anidulafungin, which undergoes spontaneous degradation [17]. Concerns about possible hepatotoxicity of micafungin have been raised which may affect its use in daily practice [2]. Two randomized controlled trials $[16,18]$ and one retrospective observational trial [19], compared the safety and efficacy of micafungin versus caspofungin in the treatment of invasive candidiasis, showing similar results in the safety and efficacy of micafungin compared with caspofungin. One systematic review including 8,000 patients [20] and one observational cohort study with 8,696 patients [21], evaluated the safety of micafungin versus other echinocandins, showing no increased risk of hepatic injury by micafungin. Both studies only evaluated the safety concerning hepatotoxicity of antifungal medication in mostly non-ICU patients, but did not evaluate mortality or efficacy.

As far as we are aware there are no studies comparing the efficacy and safety of micafungin versus anidulafungin in critically ill patients with invasive candidiasis. We performed a retrospective study to compare the safety and efficacy of micafungin versus anidulafungin in critically ill patients with invasive candidiasis.

\section{Methods}

\section{Patients}

We retrospectively gathered data from the patients' medical records using a predefined checklist. Between January 1, 2012, and January 1, 2015, all patients over the age of 18 with invasive candidiasis and who only received anidulafungin or micafungin as systemic antifungal treatment in the Intensive Care Unit (ICU) of the Erasmus University Medical Center Rotterdam, were considered for participation. Candidemia was defined as at least one positive blood culture for Candida spp. drawn from a peripheral vein. Invasive candidiasis was defined as a positive culture with Candida spp. obtained from a normally sterile site, such as pleural or peritoneal fluid, in the context of pleural exsudate/empyema, and secondary or tertiary peritonitis following a ruptured viscus and surgery, respectively [22]. In addition, patients needed to have one or more of the following signs and symptoms of infection: fever or hypothermia; hypotension; localized signs and symptoms of inflammation; or radiological findings of invasive candidiasis. Between January 2012 and December 2014 there were 124 patients diagnosed with invasive candidiasis of whom 20 received caspofungin, 38 stepped-down to fluconazole and 3 received both antifungals. In total there were 63 patients with invasive Candida spp. infection who only received anidulafungin or micafungin. The Dutch law states that informed consent is not required in case of retrospective analysis in which data collected during routine clinical care were used and anonymously analyzed.

\section{Clinical protocol}

Patients were taken care of by attending intensivists according to national and local guidelines. In our center selective decontamination of the digestive tract (SDD) is routinely used for patients with an expected duration of mechanical ventilation for more than $48 \mathrm{~h}$. This involves administration of an oral paste and of a suspension via the nasogastric tube, containing the non-absorbable antibiotics tobramycin, amphotericin-B and colistin. Patients also received cefotaxime intravenously at 4 times $1 \mathrm{~g}$ a day for a three-day period. Inventory cultures are taken of the throat, tracheal aspirates, and rectum as part of this protocol on admission. To monitor the effect of SDD treatment, surveillance cultures (from throat, tracheal aspirates, and rectum) were routinely performed three times per week. All SDD cultures were screened for the presence of Gram-negative rods, and yeast that were identified to the species level (see below). In case a patient is suspected for having an infection, additional cultures (besides the routinely SDD cultures) can be taken from the possible source of infection, which includes the use of blood cultures. All materials obtained from a normally sterile site were cultured onto relevant agar plates for the detection of both bacteria and yeast. Blood culture bottles, specific for the recovery of yeast (BACTEC Mycosis IC/F), that became positive were subcultured onto chocolate agar, Sabouraud agar, and CHROMagar ${ }^{\mathrm{TM}}$ to ensure purity or mixed infection and differentiation of yeast, and incubated at $35{ }^{\circ} \mathrm{C}$. Auxacolor (Sanofi Diagnostics Pasteur) or MALDI-TOF was used to identify the species level of a Candida colony as soon as visible growth from a normally sterile site was observed. Susceptibility testing of isolates obtained from normally sterile sites was performed using a CLSI broth microdilution method (Sensititre ${ }^{\circ}$, Thermoscientific, USA) and results for azoles, amphotericin B and caspofungin, were reported according to revised speciesspecific CLSI clinical breakpoints. For fluconazole, $C$. albicans was considered susceptible if the minimum inhibitory concentration (MIC) was $\leq 2 \mathrm{mg} / \mathrm{L}$, and reduced susceptibility was defined as a MIC of $\geq 4 \mathrm{mg} / \mathrm{L}$ [23], after $24-48 \mathrm{~h}$ of growth. The decision to start an echinocandin was taken by the attending intensivist in collaboration with infectious disease specialist. The initiation of antifungal therapy was based on clinical signs (i.e. fever, hypothermia, hypotension, leukocytosis or leukopenia), risk factors for invasive candidiasis, culture results, radiological findings of invasive candidiasis, and 
according to published criteria [9]. Caspofungin was introduced in 2001, followed by anidulafungin and micafungin respectively. In case of severe liver injury and liver failure, which was defined as the presence of clinical signs and symptoms of an abnormal liver function (increased liver enzymes, hyperbilirubinemia, coagulopathy and encephalopathy), intensivists prefer anidulafungin over micafungin. In all other instances, the choice between anidulafungin or micafungin was at the discretion of the attending intensivist. For anidulafungin patients received an intravenous daily dose of $100 \mathrm{mg}$ after an initial single loading dose of $200 \mathrm{mg}$. Micafungin was administered at a dose of $100 \mathrm{mg}$ intravenous once daily without a loading dose. No dose adjustment was needed for body weight or impaired renal or hepatic function. Drainage of suspected pus collections as well as removal of IV catheters suspected to be the origin of infection was routinely done. The duration of the antifungal therapy was decided in close collaboration with the infectious diseases physician, based on Dutch invasive fungal infection guidelines which take several factors into account, such as duration of positive cultures, the certainty of good drainage and clinical improvement (absence of fever for $>24 \mathrm{~h}$, haemodynamic stability, and neutropenia) $[9,24,25]$.

\section{Study protocol and data collection}

Demographic data and clinical date were recorded on admission, including severity of illness scores, risk factors for invasive Candida spp. infection (neutropenia, recent surgery, diabetes mellitus, cancer, mechanical ventilation, renal replacement therapy, total parenteral nutrition, transplant and central venous catheters), the duration of ICU stay, and mortality at day 28 and 90 after start of antifungal therapy. Patients were checked for abnormal liver function during antifungal therapy, serum aspartate transaminase (AST) and serum alanine transferase (ALT) were recorded at start and stop of echinocandin treatment. Serum bilirubin, prothrombin time (PT), AST, and ALT peak values were recorded daily during echinocandin treatment. At the start of echinocandin treatment a Candida score was calculated for each patient to help, when $>3$, establishing the risk for invasive candidiasis vs colonization [26]. The primary site of infection and the obtained culture results were recorded. A global response at the end of treatment was defined as both clinical success (cure - resolution of signs and symptoms of Candida spp. infection, or improvement - incomplete resolution of signs and symptoms of Candida spp. infection) and microbiological success (2 negative follow-up cultures for Candida spp., or presumed eradication when the follow-up culture was not available and clinical response was defined as cure or improvement at the end of treatment). The duration of Candida spp. infection was defined as the period between the first positive culture and the first negative culture or ICU death or discharge. Duration of treatment was defined as the period between initiation and stop of antifungal therapy or ICU death or discharge.

\section{Statistical analysis}

Continuous variables were presented with median and interquartile range because of the not normal distribution (Kolmogorov-Smirnov test, $P<0.05$ ). Continuous data were examined with The Mann-Whitney $U$ test and categorical data with the Fisher exact test. The Kaplan-Meier estimation of survival curves (compared by log rank tests) and multivariable cox regression were used for survival analysis. All reported $P$ values are two-tailored. Statistical significance was set at $P<0.05$.

\section{Results}

Sixty-three patients with invasive candidiasis received either anidulafungin $(n=30)$ or micafungin $(n=33)$. Patients using anidulafungin were more often on renal replacement therapy (Table 1 ).

\section{Infection and treatment characteristics}

Of the 63 patients, 22 patients had candidemia, 37 patients had abdominal and 4 patients had pleural infection (Table 2). The average Candida score was 3 for both groups. Anidulafungin was initiated at median day 3 and micafungin was initiated at median day 2 after ICU admission. There were no echinocandin-resistant strains. Four $C$. albicans isolates were considered less sensible to fluconazole with an average clinical breakpoint of $100 \mathrm{mg} / \mathrm{L}$. There was no difference in the duration of treatment and infection, and response rates, even when corrected for liver failure. Both groups had a similar duration of ICU stay. No difference was observed in 28-day mortality, but 90-day mortality was higher in patients on anidulafungin (Table 2 and Fig. 1). Multivariable Cox regression analysis showed that age (hazard ratio $1.13,95 \%$ confidence interval $1.02-1.23, P=0.02$ ) and serum bilirubin (hazard ratio 1.06, $95 \%$ confidence interval $1.01-1.12, P=0.03$ ) were the best parameters for the prediction of 90-day mortality, whereas APACHE II, Candida score and antifungal therapy did not contribute $(P>0.05)$.

\section{Liver enzymes and function}

Patients with invasive candidiasis and liver failure were more often treated with anidulafungin than micafungin. None of the patients in this study developed liver failure or elevated liver enzymes requiring 
Table 1 Baseline demographic and clinical characteristics

\begin{tabular}{|c|c|c|c|}
\hline & $\begin{array}{l}\text { Anidulafungin } \\
(n=30)\end{array}$ & $\begin{array}{l}\text { Micafungin } \\
(n=33)\end{array}$ & $P$ \\
\hline \multicolumn{4}{|l|}{ On admission } \\
\hline Age (years) & $59(20)$ & $62(20)$ & 0.36 \\
\hline Gender (male) ${ }^{\mathrm{a}}$ & $19(63)$ & $26(79)$ & 0.18 \\
\hline APACHE II score & $26(10)$ & $23(8)$ & 0.13 \\
\hline SOFA score & $10(9)$ & $9(3)$ & 0.63 \\
\hline Reasons of ICU admission ${ }^{a}$ & & & 0.14 \\
\hline Suspected sepsis & $9(30)$ & $11(33)$ & \\
\hline Respiratory failure & $2(7)$ & $6(19)$ & \\
\hline Renal failure & $1(3)$ & $0(0)$ & \\
\hline Liver failure & $4(13)$ & $0(0)$ & \\
\hline CPR & $2(7)$ & $2(6)$ & \\
\hline Shock & $5(17)$ & $3(10)$ & \\
\hline Postoperative & $7(23)$ & $11(34)$ & \\
\hline \multicolumn{4}{|l|}{ At start of echinocandin } \\
\hline \multicolumn{4}{|l|}{ Risk factors for invasive candidiasis ${ }^{a}$} \\
\hline Neutropenia & $3(10)$ & $2(6)$ & 0.57 \\
\hline Broad spectrum antibiotics & $18(60)$ & $15(45)$ & 0.25 \\
\hline Immunosuppression & $4(13)$ & $3(9)$ & 0.60 \\
\hline Steroids & $16(53)$ & $12(36)$ & 0.18 \\
\hline TPN & $4(13)$ & $11(33)$ & 0.07 \\
\hline Recent Surgery & $8(27)$ & $15(45)$ & 0.13 \\
\hline DM ॥ & $11(37)$ & $7(21)$ & 0.18 \\
\hline Malignancy & $5(17)$ & $11(33)$ & 0.13 \\
\hline Transplant & $5(17)$ & $4(12)$ & 0.61 \\
\hline $\mathrm{CWH}$ & $19(63)$ & $7(21)$ & 0.001 \\
\hline CVC & $29(97)$ & $29(88)$ & 0.20 \\
\hline Mechanical ventilation & $27(90)$ & $32(97)$ & 0.26 \\
\hline $\begin{array}{l}\text { Duration between admission and } \\
\text { start echinocandin (days) }\end{array}$ & $3(4)$ & $2(2)$ & 0.17 \\
\hline
\end{tabular}

Numbers (percentage) a or median (interquartile range), where appropriate List of abbreviations: APACHE II Acute Physiology and Chronic Health Evaluation II, CPR cardiac pulmonary resuscitation, $C V V H$ continuous venovenous haemofiltration, CVC central venous catheter, DM II diabetes mellitus type II, ICU intensive care unit, TPN total parenteral nutrition

cessation of treatment related to the use of an echinocandin. PT, AST, ALT and bilirubin levels tended to be higher in patients treated with anidulafungin. When excluding liver failure, no differences were seen in PT, AST, ALT and bilirubin levels between the groups (Table 3 ).

\section{Costs}

Table 4 describes the involved costs for both treatment strategies. The total treatment costs per patient seemed to be higher for micafungin, because of the longer treatment period. However, the treatment costs per patient per day were lower for micafungin.
Table 2 Infection and treatment characteristics

\begin{tabular}{|c|c|c|c|}
\hline & $\begin{array}{l}\text { Anidulafungin } \\
(n=30)\end{array}$ & $\begin{array}{l}\text { Micafungin } \\
(n=33)\end{array}$ & $P$ \\
\hline Candida score at start & $3(2)$ & $3(3)$ & 0.71 \\
\hline Source of isolates ${ }^{a}$ & & & 0.25 \\
\hline Blood & $10(33)$ & $12(36)$ & \\
\hline Intra-abdominal fluid & $17(57)$ & $20(63)$ & \\
\hline Pleural fluid & $3(10)$ & $1(3)$ & \\
\hline Candida spp. ${ }^{a}$ & & & 0.46 \\
\hline Candida albicans & $8(27)$ & $3(9)$ & \\
\hline Candida dublienis & $0(0)$ & $2(6)$ & \\
\hline Candida glabrata & $17(57)$ & $21(66)$ & \\
\hline Candida krusei & $3(10)$ & $3(10)$ & \\
\hline Candida parapsilosis & $1(3)$ & $2(6)$ & \\
\hline Candida tropicalis & $1(3)$ & $2(6)$ & \\
\hline Global response ${ }^{a}$ & $21(67)$ & $23(70)$ & 0.80 \\
\hline Clinical response $^{a}$ & $24(80)$ & $28(85)$ & 0.62 \\
\hline Microbial response ${ }^{a}$ & $21(70)$ & $24(73)$ & 0.81 \\
\hline Gaining negative cultures ${ }^{a}$ & $18(60)$ & $20(60)$ & 0.96 \\
\hline Duration of infection (days) & $3(5)$ & $3(5)$ & 0.80 \\
\hline Duration of treatment (days) & $12(8)$ & $14(9)$ & 0.40 \\
\hline Length of ICU stay (days) & $13(15)$ & $14(21)$ & 0.64 \\
\hline Mortality day 28 after start ${ }^{a}$ & $20(67)$ & $18(55)$ & 0.33 \\
\hline Mortality day 90 after start ${ }^{a}$ & $26(87)$ & $21(64)$ & 0.04 \\
\hline
\end{tabular}

Numbers (percentage) ${ }^{a}$ or median (interquartile range), where appropriate Abbreviations: ICU intensive care unit

\section{Discussion}

This study suggests that in critically ill patients with candidemia or invasive candidiasis, the safety and efficacy of the treatment with micafungin was similar to that of anidulafungin. No differences were seen in response rates, liver function and enzymes, and mortality. Treatment with micafungin seems to be less expensive then that with anidulafungin.

Echinocandins inhibit synthesis of the $\beta-(1-3)-D-$ glucan compound of the fungal cell wall and are considered as safe drugs [2, 17]. The overall efficacy between the three echinocandins is comparable, showing only little difference [27]. Nevertheless, the EMA still recommend to only use micafungin in case other antifungals are not appropriate, as rat experiments suggested a potential risk for the development of liver tumors [27]. However, these results were obtained from studies using high dosages for prolonged time in male animals, similar effects were not reported by other studies on humans or animals [17]. Both caspofungin and micafungin undergo hepatic metabolization, in contrast to anidulafungin, which undergoes spontaneous degradation [17]. Transient elevation of liver enzymes occurs in 2 to $15 \%$ of patients treated 


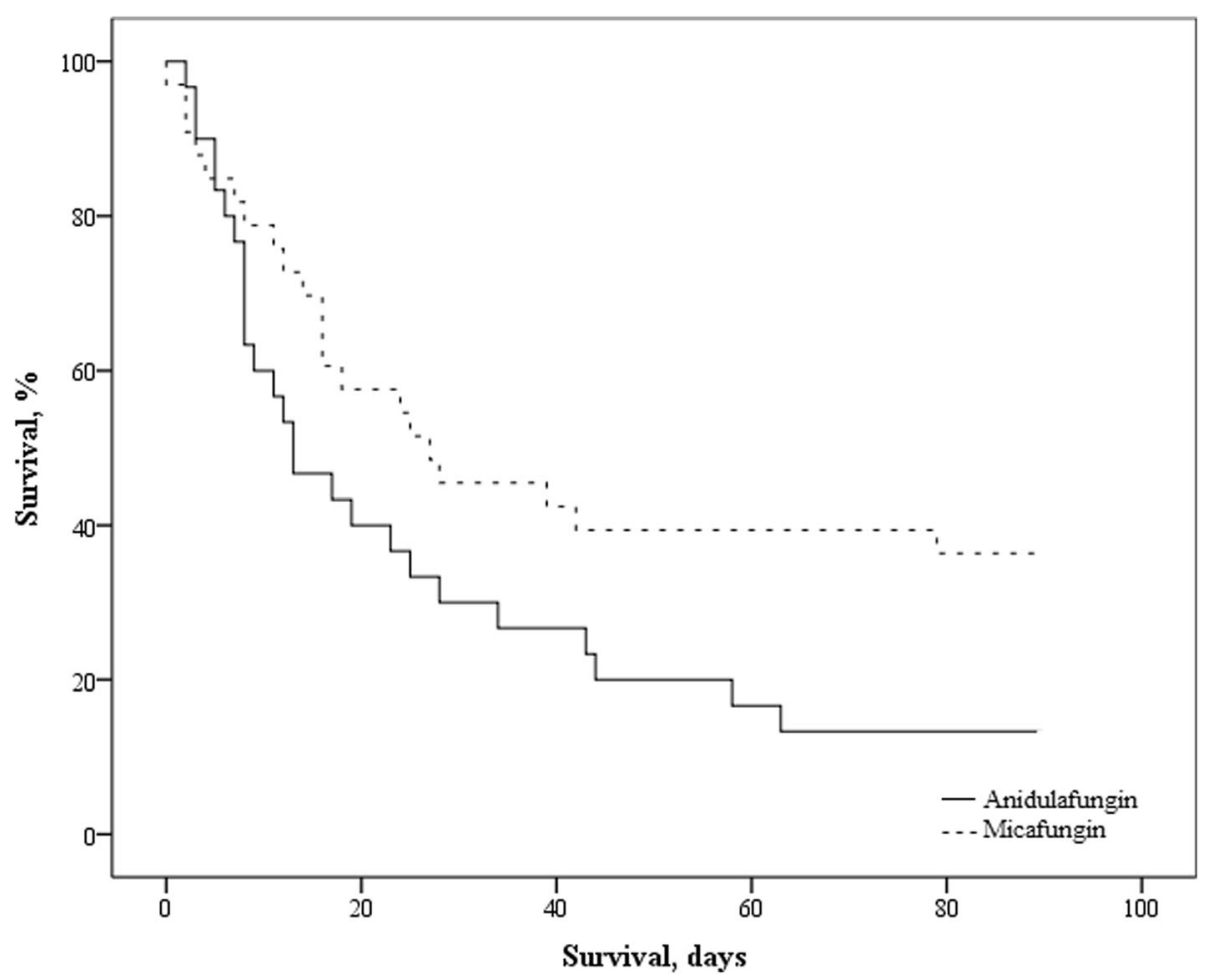

Fig. 1 Kaplan-Meier survival curve up to day 90 after initiation of an echinocandin, $P=0.04$ (log rank test). Numbers at risk, micafungin group: 33 , $19,14,13,12$. Numbers at risk, anidulafungin group: $30,12,8,5,4$

with an echinocandin [28]. In this study we found an elevation in serum AST of $38 \%$ and $35 \%$, and an elevation in serum ALT of $33 \%$ and $29 \%$, in patients treated with anidulafungin or micafungin, respectively. The observed incidence of elevated liver enzymes in this study is much higher, but equal between both groups of echinocandins, but we only looked at elevated liver enzymes in general and not specifically caused by the echinocandins. Abnormal liver function tests can be found in up to $61 \%$ of critically ill patients, as caused by sepsis, drugs or ischemia [29]. Our results suggest that micafungin is as safe as anidulafungin concerning hepatotoxicity. The results are in line with two previous studies, which both concluded that anidulafungin and micafungin had a low risk of elevated liver enzyme levels not requiring the cessation of treatment $[20,21]$. Both studies only evaluated the safety concerning hepatotoxicity of antifungal medication in mostly non-ICU patients, but did not evaluate mortality or efficacy, as we did. Therefore, this study adds important information about the safety and efficacy of micafungin compared with anidulafungin in critically ill patients. At day 28 the overall mortality was $60 \%$ which is comparable to the mortality described in other studies [30, 31]. The data suggest that higher 90-day mortality with anidulafungin than micafungin reflects more severe underlying liver disease rather than effect of treatment itself.

The type and duration of treatment of invasive candidiasis depends on culture results and sensitivity testing, the extent of organ involvement and patients' clinical condition $[9,24]$. In patients with invasive fungal infections the recommended length of therapy is 14 days after the first negative blood culture $[9,24]$, which is in line with the observed median length of treatment of 12-14 days in this study. The overall response rates in this study are comparable to those found in other performed studies [11,32]. The overall response rate of micafungin is comparable to that of anidulafungin. Our retrospective data, concerning the safety and efficacy of micafungin are in line with a recent performed study, which evaluated the safety and efficacy of micafungin monotherapy in critically ill patients with cancer and invasive candidiasis [32]. However, the study did not make a comparison with anidulafungin, as we did. The pharmacokinetics of micafungin are very well defined in noncritically ill patients and seems to be similar in critically ill patients [33]. In critically ill patients micafungin reaches a steady state by day 3 , without the need for a loading dose, in contrast to caspofungin and anidulafungin $[9,24,25,33]$. Dose adaptations are not required for body weight and in patients with renal or hepatic impairment and renal replacement therapy [33]. The 
Table 3 Liver enzymes and function

\begin{tabular}{|c|c|c|c|}
\hline A & $\begin{array}{l}\text { Anidulafungin } \\
(n=30)\end{array}$ & $\begin{array}{l}\text { Micafungin } \\
(n=33)\end{array}$ & $P$ \\
\hline Liver failure $^{a}$ & $9(30)$ & $2(6)$ & 0.01 \\
\hline $\begin{array}{l}\text { Increased serum AST (U/L) } \\
\text { after start }^{\mathrm{a}}\end{array}$ & $13(43)$ & $12(36)$ & 0.58 \\
\hline $\begin{array}{l}\text { Increased serum ALT (U/L) } \\
\text { after start }^{\mathrm{a}}\end{array}$ & $13(43)$ & $10(30)$ & 0.29 \\
\hline Serum AST (U/L) at start & $95(282)$ & $55(76)$ & 0.09 \\
\hline Serum ALT (U/L) at start & $56(125)$ & $39(59)$ & 0.13 \\
\hline Serum AST (U/L) at stop & $115(1011)$ & $52(108)$ & 0.17 \\
\hline Serum ALT (U/L) at stop & 75 (312) & $45(68)$ & $c$ \\
\hline Serum AST (U/L) peak & $285(228)$ & $116(181)$ & 0.11 \\
\hline Serum ALT (U/L) peak & $150(404)$ & $70(126)$ & 0.15 \\
\hline Serum bilirubin ( $\mu \mathrm{mol} / \mathrm{L})$ peak & $71(192)$ & $15(29)$ & 0.05 \\
\hline Prothrombin Time (sec) peak & $18.3(15.4)$ & $17.8(8.0)$ & 0.27 \\
\hline B & $\begin{array}{l}\text { Anidulafungin } \\
(n=21)\end{array}$ & $\begin{array}{l}\text { Micafungin } \\
(n=31)\end{array}$ & $P$ \\
\hline $\begin{array}{l}\text { Increased serum AST (U/L) } \\
\text { after start }^{\mathrm{a}}\end{array}$ & $8(38)$ & $11(35)$ & 0.85 \\
\hline $\begin{array}{l}\text { Increased serum ALT (U/L) } \\
\text { after start }^{\mathrm{a}}\end{array}$ & $7(33)$ & $9(29)$ & 0.74 \\
\hline Serum AST (U/L) at start & $61(98)$ & $55(73)$ & 0.82 \\
\hline Serum ALT $(U / L)$ at start & $43(75)$ & $39(54)$ & 0.89 \\
\hline Serum AST (U/L) at stop & $44(141)$ & $49(79)$ & 0.98 \\
\hline Serum ALT $(U / L)$ at stop & $41(83)$ & $45(54)$ & 0.99 \\
\hline Serum AST (U/L) peak & $129(267)$ & $113(158)$ & 0.88 \\
\hline Serum ALT (U/L) peak & 69 (209) & $61(119)$ & .95 \\
\hline Serum bilirubin ( $\mu \mathrm{mol} / \mathrm{L})$ peak & $20(105)$ & $14(27)$ & 0.74 \\
\hline Prothrombin Time (sec) peak & $16.6(7.5)$ & $17.8(5.3)$ & 0.00 \\
\hline
\end{tabular}

Numbers (percentage) ${ }^{a}$ or median (interquartile range). Liver enzymes and function in all patients who were treated with anidulafungin and micafungin (A) and in patients without liver failure (B)

Abbreviations: ALT alanine transaminase, AST aspartate transaminase

Table 4 Treatment costs

\begin{tabular}{lll}
\hline & $\begin{array}{l}\text { Anidulafungin } \\
(n=30)\end{array}$ & $\begin{array}{l}\text { Micafungin } \\
(n=33)\end{array}$ \\
\hline Total treatment days & 354 & 429 \\
Total drug dose $(\mathrm{mg})$ & 41.600 & 42.900 \\
Total drug costs $(€)$ & $183,805.44$ & $209,553.63$ \\
Drug costs per patient $(€)$ & $6,126.84$ & $6,350.11$ \\
Drug costs p.p.p.d. $(€)$ & 519.22 & 488.47 \\
\hline
\end{tabular}

Micafungin costs $€ 460.82$ (excluding VAT) per $100 \mathrm{mg}$ ampoule, $€ 488.47$ (including $6 \%$ VAT)

Anidulafungin costs $€ 416.83$ (excluding VAT) per $100 \mathrm{mg}$ ampoule, $€ 441.84$ (including $6 \%$ VAT)

Abbreviations: p.p.p.d. per patient per day involved costs per patient per day of treatment with micafungin seems to be a bit lower compared with anidulafungin.

One of the limitations of this study is the relatively small number of patients included, so that results should be regarded as preliminary. This study enrolled 63 patients with invasive candidiasis caused by Candida spp. in a four year period in a tertiary care ICU with 2,000 admissions per year. Hence, the reported incidence of invasive candidiasis in a large review was 5 to 10 cases per 1,000 ICU admission, which is comparable with our reported incidence of 6 per 1,000 ICU admissions [34]. Second, because of the retrospective design we cannot exclude that the use of either agent may have been subject to bias.

\section{Conclusion}

In conclusion, our results suggest that micafungin can be safely and effectively used in critically ill patients with candidemia and invasive candidiasis.

\section{Abbreviations}

ALT: serum alanine transferase; APACHE II: The acute physiology and chronic health evaluation II serum aspartate transaminase; AST: Serum aspartate transaminase; EMA: European medicines agency; FDA: Food and drug administration; ICU: Intensive care unit; IV: Intravenous; MIC: Minimum inhibitory concentration; PPPD: Per patient per day; PT: Prothrombin time; SDD: Selective decontamination of the digestive tract; SOFA: Sequential organ failure assessment; SPP: Species

\section{Acknowledgements}

None to declare.

Funding

This work was not supported by any funding.

Availability of data and materials

The data and materials can be obtained on request from the authors.

\section{Authors'contribution}

PJG, ABJG, and NGMH designed the study. PJG, SL and ABJG performed the analyses. PJG, SL and NGMH collected the data. PJG and ABJG drafted the manuscript. All authors reviewed the manuscript. All Authors read and approved the final version of this manuscript.

\section{Competing interests}

The authors declare that they have no competing interests.

Consent for publication

Not applicable.

\section{Ethics approval and consent to participate}

This study does not need approval from a medical ethical committee for the following reason. Division 1, Section 1.1.b. of the Dutch law on medical research defines medical research as research in which persons are subjected to treatment or are required to follow a certain behavioral strategy (www.ccmo.nl, http://www.ccmo.nl/attachments/files/wmo-engelse-vertaling29-7-2013-afkomstig-van-vws.pdf). This means that retrospective patient data analysis is not subject to this law. Indeed, informed consent is not needed, provided that, as we did, data are retrieved and analyzed anonymously.

\section{Author details}

${ }^{1}$ Department of Intensive Care Medicine, Erasmus University Medical Center, 's Gravendijkwal 230, 3015 CE Rotterdam, The Netherlands. ²Department of 
Pharmacy, Erasmus University Medical Center, 's Gravendijkwal 230,

Rotterdam 3015 CE, The Netherlands.

\section{Received: 26 January 2016 Accepted: 9 September 2016 Published online: 15 September 2016}

\section{References}

1. Kett DH, Azoulay E, Echeverria PM, Vincent JL. Candida bloodstream infections in intensive care units: analysis of the extended prevalence of infection in intensive care unit study. Crit Care Med. 2001;39:665-70.

2. Glockner A. Treatment and prophylaxis of invasive candidiasis with anidulafungin, caspofungin and micafungin - review of the literature. Eur J Med Res. 2011:16:167-79.

3. Pelz RK, Lipsett PA, Swoboda SM, Diener-West M, Hammond JM, Hendrix $\mathrm{CW}$. The diagnostic value of fungal surveillance cultures in critically ill patients. Surg Infect. 2000;1:273-81.

4. Dimopoulos G, Ntziora F, Rachiotis G, Armaganidis A, Falagas ME. Candida Albicans versus non-candida albicans intensive care unit-acquired bloodstream infections: differences in risk factors and outcome. Anesth Analg. 2008:106:523-9.

5. Prowle GR, Echeverri JE, Ligabo EV, Sherry N, Taori GC, Crozier TM, et al. Acquired bloodstream infection in the intensive care unit: incidence and attributable mortality. Crit Care. 2011;15:R100.

6. Morrell M, Fraser VJ, Kollef MH. Delaying the empiric treatment of candida bloodstream infection until positive bloodculture results are obtained: a potential risk factor for hospital mortality. Antimicrob Agents Chemother. 2005;49:3640-5.

7. Garey KW, Rege M, Pai MP, Mingo DE, Suda KJ, Turpin RS, et al. Time to initiation of fluconazole therapy impacts mortality in patients with candidemia: a multi institutional study. Clin Infect Dis. 2006;45:25-31.

8. Pfaller M, Boyken I, Hollis RJ, Kroeger J, Messer SA, Tendolkar S, et al. In vitro susceptibility of invasive isolates of candida spp. to anidulafungin, caspofungin, and micafungin: six years of global surveillance. J Clin Microbiol. 2008:46:150-6

9. Pappas PG, Kauffman CA, Andes D, Benjamine Jr DK, Calandra TF, Edwards $\mathrm{Jr} \mathrm{JE}$, et al. Clinical guidelines for the management of candidiasis: 2009 update by the infectious diseases society of America. Clin Infect Dis. 2009;48:505-35

10. Van Burik JH, Ratanatharathorn V, Stepan DE, Miller CB, Lipton JH, Vesole $\mathrm{DH}$, et al. Micafungin versus fluconazole for prophylaxis against invasive fungal infections during neutropenia in patient undergoing hematopoietic stem cell transplantation. Clin Infect Dis. 2004;39:1407-16.

11. Reboli AC, Rotstein CM, Pappas PG, Chapman SW, Kett DH, Kumar D, et al. Anidulafungin versus fluconazole for invasive candidiasis. N Engl J Med. 2007:356:2472-82.

12. Hiramatsu Y, Maeda Y, Fuji N, Saito T, Nawa Y, Hara M, et al. Use of micafungin versus fluconazole for antifungal prophylaxis in neutropenic patients receiving hematopoietic stem cell transplantation. Int J Hematol. 2008;88:588-95

13. Guery BP, Arendrup MC, Auzinger G, Azoulay E, Borges Sá M, Johnson EM, et al. Management of invasive candidiasis and candidemia in adult nonneutropenic intensive care unit patients: Part II treatment. Intensive Care Med. 2009:35:206-14

14. Marchetti $O$, Eggimann $P$, Calandra T. Invasive candidiasis in critically ill patients: does progressing knowledge improve clinical management and outcome? Curr Opin Crit Care. 2010;16:442-4.

15. Kett DH, Shorr AF, Reboli AC, Reisman AL, Biswas P, Schlamm HT. Anidulafungin compared with fluconazole in severely ill patients with candidemia and other forms of invasive candidiasis: support for the 2009 IDSA treatment guidelines for candidiasis. Crit Care. 2011;15:R253.

16. Pappas PG, Coleman M, Rotstein F, Nucci M, Talwar D, De Waele JJ, et al. Micafungin versus caspofungin for treatment of candidemia and other forms of invasive candidiasis. Clin Infect Dis. 2007;45:883-93.

17. Dimopoulos G, Antonopoulou A, Armaganidis A, Vincent JL. How to select an antifungal agents in critically ill patients. J Crit Care. 2013;28:717-27.

18. Kubiak DW, Bryar JM, McDonnell AM, Delgado-Flores JO, Mui E, Baden LR, et al. Evaluation of caspofungin or micafungin as empiric antifungal therapy in adult patients with persistent febrile neutropenia: a retrospective, observational, sequential cohort analysis. Clin Ther. 2010;32:637-48.

19. Kohno S, Izumikawa K Yoshida M, Takesue Y, Oka S, Kamei K, et al. A double-blind comparative study of the safety and efficacy of caspofungin versus micafungin in the treatment of candidiasis and aspergillosis. Eur J Clin Microbial Infect Dis. 2013:32:387-97.

20. Wang $J$, Chang $\mathrm{CH}$, Young-Xu Y, Chan KA. Systematic review and metaanalysis of the tolerability and hepatotoxicity of antifungals in empirical and definitive therapy for invasive fungal infection. Antimicrob Agents Chemother. 2010:54:2409-19.

21. Schneeweiss S, Galar A, carver $P$, Johnson M, Marty F, Nagel J, et al. Multicenter cohort study of the safety of Micafungin and other parenteral antifungals. ICAAC-ICC 2015; poster presentation M-364.

22. Bassetti M, Marchetti M, Chakrabarti A, Colizza S, Garnacho-Montero J, Kett $\mathrm{DH}$, et al. A research agenda on the management of intra-abdominal candidiasis: results from a consensus of multinational experts. Intensive Care Med. 2013:39:2092-106.

23. Vazquez J, Reboli AC, Pappas PG, Patterson TF, Reinhardt J, Chin-Hong P, et al. Evaluation of an early step-down strategy from intravenous anidulafungin to oral azole therapy for the treatment of candidemia and other forms of invasive candidiasis: results from an open-label trial. BMC Inf Dis. 2014;14:97.

24. Cornely OA, Bassetti M, Calandra T, Garbino J, Kullberg BJ, Lortolary O, et al. ESCMID guideline for the diagnosis and management of Candida diseases 2012: non-neutropenic adult patients. Clin Microbiol Inf. 2012;18:19-37.

25. Oude Lashof AML, Janssen JJWM, Meis JFGM, Warris A, van 't Wout JW, Natsch SS, et al. SWAB- richtlijn voor de behandeling van invasieve schimmelinfecties 2013. NVMM. 2013:18:13-7.

26. León C, Ruiz-Santana S, Saavedra P, Galván B, Blanco A, Castro C, et al. Usefulness of the 'Candida score' for discriminating between Candida colonization and invasive candidiasis in non-neutropenic critically ill patients: A prospective multicenter study. Crit Care Med. 2009;37(367):1624-33.

27. Kullberg BJ, Verweij PE, Akova M, Arendrup MC, Bille J, Calandra T, et al. European expert opinion on the management of invasive candidiasis in adults. Clin Microbial Inf. 2011;5:1-12.

28. Cappelletty D, Eiselstein-McKitrick K. The echinocandins. Pharmacotherapy. 2007;27:369-88

29. Thomson SJ, Cowan L, Johnston I, et al. Liver function tests on the intensive care unit: a prospective, observational study. Intensive Care Med. 2009;35: 1406-11.

30. Guery BP, Arendrup MC, Auzinger G, Azoulay E, Borges Sa M, Johnson EM, et al. Management of invasive candidiasis and candidemia in adult nonneutropenic intensive care unit patients: Part I. Epidemiology and diagnosis. Intensive Care Med. 2009;35:55-62.

31. Leroy $\mathrm{O}$, Gangneux JP, Montravers P, Mira JP, Gouin F, Sollet JP, AmarCand study group, et al. Epidemiology, management, and risk factors for death of invasive Candida infections in critical care: a multicenter, prospective, observational study in France (2005-2006). Crit Care Med. 2009:309(37):1612-8.

32. Farmakiotis D, Tarrand JJ, Kontoyiannis DP. Efficacy and tolerability of micafungin monotherapy for candidemia and deep-seated candidiasis in adults with cancer. Antimicrob Agents Chemother. 2014;58:3526-9.

33. Lempers VJ, Schouten JA, Hunfeld NG, Colbers A, Van Leeuwen HJ, Burger DM, et al. Altered micafungin pharmacokinetics in intensive care unit patients. Antimicrob Agents Chemother. 2015;59:4403-9.

34. Eggimann P, Bille J, Marchetti O. Diagnosis of invasive candidiasis in the ICU. Ann Intensive Care. 2011;1:37.

\section{Submit your next manuscript to BioMed Central and we will help you at every step:}

- We accept pre-submission inquiries

- Our selector tool helps you to find the most relevant journal

- We provide round the clock customer support

- Convenient online submission

- Thorough peer review

- Inclusion in PubMed and all major indexing services

- Maximum visibility for your research

Submit your manuscript at www.biomedcentral.com/submit 